\title{
The GONet (Ground Observation Network) Camera: An Inexpensive Light Pollution Monitoring System
}

\author{
Ken WALCZAK ${ }^{1 *}$, Grace CRIM ${ }^{1}$, Thane GESITE ${ }^{1}$, Salome HABTEMICHAEL ${ }^{1}$, Jack \\ MORGAN $^{1}$, Cynthia TARR ${ }^{1}$, Laris TURKIC $^{1}$, and Jeff WIEDEMANN ${ }^{1}$
}

${ }^{1}$ Adler Planetarium, Chicago, Illinois 60605, USA

Received 18 September 2020, Accepted 16 April 2021

\begin{abstract}
Instrumentation developed to monitor and characterize light pollution from the ground has helped frame our understanding of the impacts of artificial light at night (ALAN). All-sky imaging has been used to quantify and characterize ALAN in a variety of environments. Over the past decade growth in access to Do-It-Yourself electronics has afforded the opportunity for the development of new and inexpensive instrumentation for ALAN research. The GONet (Ground Observing Network) camera is an inexpensive ( USD 100), simple to use, all-sky imaging system designed to allow measurements of sky quality at night. Due to their ease of use and low price, GONet cameras allow observations by users with little technical expertise, large inter-comparison campaigns and deployments of opportunity. Developed as a student engineering project at the Adler Planetarium, initial field tests of the GONet system have demonstrated its utility as a tool that can benefit ALAN research. Here we present an overview of the design and use of the GONet device, methods of calibration, initial results from observations, potential use cases, and limitations of the system. What we describe here is the version 1 GONet camera. We conclude with a brief description of the version 2 unit already under development.
\end{abstract}

Keywords: Light Pollution, Instrumentation, All-sky imaging, Sky brightness, Monitoring

\section{Rationale}

Our proper understanding of how artificial light at night (ALAN) impacts the nighttime environment on Earth relies on data capable of characterizing it. To develop a clear picture of the diverse phenomenon resulting from ALAN, ground based sensing of light at night is critical. A number of instruments have been used to collect data on the quantity and form of light at night in a range of environments [1-4]These instruments vary from inexpensive single point sensors [5,6], off-the-shelf digital cameras equipped with fish-eye lenses[7], to custom built systems with a range of cost and capabilities[8]. Although each of these devices or systems offer particular benefits to research, we perceived a gap in the availability of an instrument capable of all-sky, color imaging designed with ease of use and at a reasonable cost. We believe a device designed with these goals in mind could help increase the data available for research and expand the potential for ground-based experiments.

The growth in the field of Do-It-Yourself (DIY) electronics in recent years has made components for the design of custom instrumentation easily accessible. Open source and shared online resources support development across this DIY community. Inexpensive GPS, sensors and imaging units are now readily available to consumers and developers. A wide range of small microcontrollers and computers such as various Arduino, Teensy, and Raspberry Pi models have been used for research and citizen science projects with user-generated code and support. Additionally, cloudbased technologies have enabled wider sharing and distribution of potentially valuable research data. The ability to

${ }^{*}$ K. Walczak, E-mail address: kwalczak@adlerplanetarium.org 
design instruments for research-specific inquiry has never been so available to such a broad audience. Recognizing the potential of these technologies for educational experiences in science and engineering, the Far Horizons program at the Adler Planetarium designed a student program based on the development of a light pollution monitoring system called GONet, the Ground Observation Network.

The initial motivation for the GONet camera, the hardware at the heart of the network, was to create a prototype device as a student STEM (Science, Technology, Engineering, Math) project to enhance observations from the NITELite (Night Imaging of Terrestrial Environments Lite) high-altitude balloon-borne light pollution imaging missions from the ground. The challenge was to design a device that could monitor night sky conditions throughout a night, by providing color, all-sky imagery, and all for a cost of about $\$ 100$. These goals were chosen because of an observed lack of instrumentation available for research between single-point systems such as the Sky Quality Meter (SQM) and costlier all-sky imagery from Digital Single Lens Reflex (DSLR) cameras and other custom imaging systems.

A comparison of the specifications and output of the GONet cameras and other ALAN monitoring instruments is outlined in Table 1. Of the single-point systems, the SQM is a standard in the ALAN research field, primarily due to its simplicity of use, relatively low cost, and per-unit calibration[9-11]. Another example of a single-point system, the TESS-W instrument, improves on the SQM with better spectral response and a shared data platform[5]. DSLR systems provide expanded data products with high resolution, color (RGB) information, and all-sky images[7,12]. Calibration with these all-sky imaging systems is possible with internal (ex., the National Park Service (NPS) system)[8] or 3rd party (ex., DiCaLum, Sky Quality Camera) software[13].

Table 1: Comparison of Specifications Among Various ALAN Sensing Instruments

\begin{tabular}{|c|c|c|c|c|c|}
\hline & SQM-L & TESS-W & GONet v1 & Fisheye DSLR & NPS \\
\hline Field of View & $20^{\circ} \mathrm{FWHM}$ & $17^{\circ} \mathrm{FWHM}$ & $2 \pi$ & $2 \pi$ & $2 \pi$ \\
\hline Resolution & $1-\mathrm{D}$ & $1-\mathrm{D}$ & $1900 \mathrm{px} / 180^{\circ}$ & $\geq 3000 \mathrm{px} / 180^{\circ}$ & $6923 \mathrm{px} / 180^{\circ}$ \\
\hline Cost & $\$ 135-\$ 300$ & $\$ 300$ & $\$ 100$ & Est. $\$ 4,500$ & $\$ 30,000$ \\
\hline Quantity & Mass produced & $\begin{array}{l}\text { Small production } \\
\text { runs }\end{array}$ & $\begin{array}{l}\text { Multiple small } \\
\text { production runs }\end{array}$ & $\begin{array}{l}\text { Commercial Off-The- } \\
\text { Shelf lens/camera }\end{array}$ & One-off design \\
\hline Size/Weight & $\begin{array}{l}8 \times 8 \times 2 \mathrm{~cm} \\
100 \mathrm{~g}\end{array}$ & $\begin{array}{c}6 \times 4 \times 4 c m \\
100 \mathrm{~g}\end{array}$ & $\begin{array}{l}12 \mathrm{x} 8 \mathrm{x} 4 \mathrm{~cm} \\
300 \mathrm{~g}\end{array}$ & $\begin{array}{l}\text { Varies } \\
1-2 \mathrm{~kg}\end{array}$ & $\begin{array}{l}\text { Large } \\
30 \mathrm{~kg}\end{array}$ \\
\hline Ease of Use & $\begin{array}{l}\text { Simple - point } \\
\text { and sample }\end{array}$ & $\begin{array}{l}\text { Simple w/ Install } \\
\text { and setup }\end{array}$ & $\begin{array}{l}\text { Simple w/ Install } \\
\text { and setup }\end{array}$ & $\begin{array}{l}\text { Requires basic } \\
\text { photography skills }\end{array}$ & $\begin{array}{l}\text { Requires technical } \\
\text { training }\end{array}$ \\
\hline
\end{tabular}

Although the simplicity of single-point systems makes rapid and long-term ALAN observation easier, they do not provide data with spatial definition, color, or multivariable information such as the evolution of contributing sources throughout a night or over time. Color information is an increasingly important aspect of ALAN characterization and understanding[14]. DSLR systems provide much richer data than single-point systems, yet a number of factors limit their potential for research. Due to the high cost of these systems widespread use is limited, obtaining numerous simultaneous observations is prohibitive as are long duration observations where a user is required to protect or monitor the equipment.

\section{Design}

The GONet camera (Error! Reference source not found.) is a small, custom-made imaging system designed for single, night-long deployments. The focus of the overall design is on simplicity of use and low cost, while still providing scientifically relevant data. 


\subsection{Structure}

The enclosure of the GONet unit is a custom case designed using Fusion 360 CAD software and is 3D printed in PLA material. This allows for complete customization of the unit, making it adaptable to the specific components used in the design as well as being rapidly reproducible. The cover is printed in opaque black PLA to prevent any interference from internal component lights. The base of the unit is printed in white, which is relatively translucent. This allows the user to monitor internal LEDs, which provide information about charging, GPS, and other statuses from components within the camera.

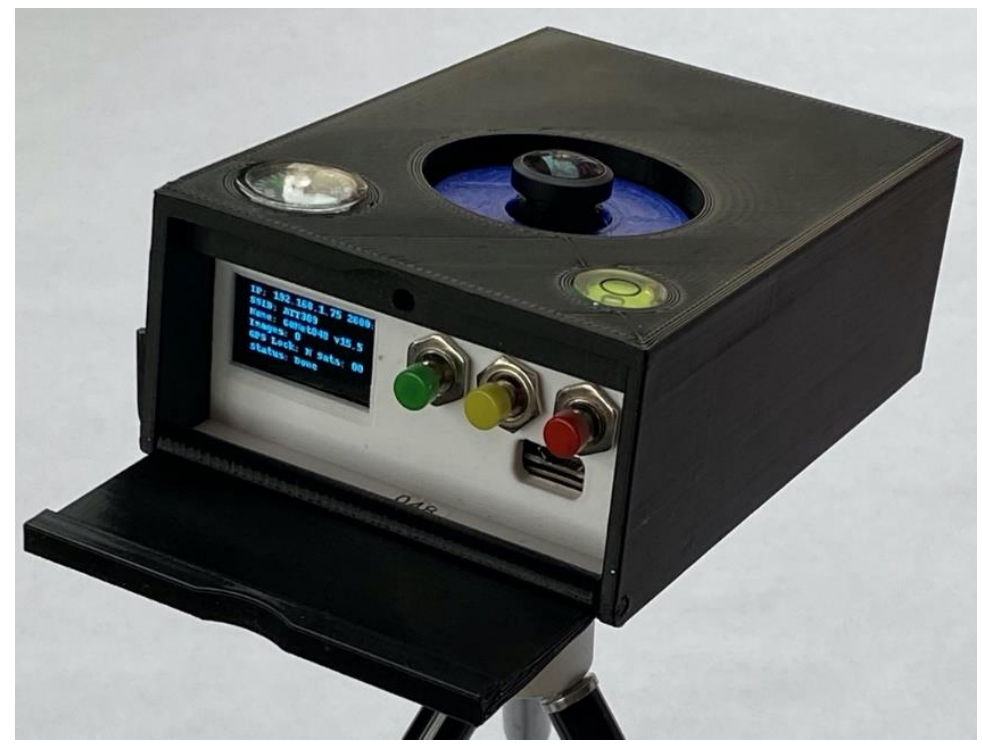

Figure 1: Version 1 Ground Observing Network (GONet) camera

Built into the cover is a spirit level for horizontally leveling the camera and a small compass to assist in orienting the unit to cardinal North. Due to internally generated magnetic fields, the compass was discovered to be inoperable and alignment is recommended with a remote compass such as one in a smart phone. The front panel door used to cover the user interface during imaging is hinged with an Allen wrench, which is removable for use in accessing the internal components. The base is equipped with a standard $1 / 4-20$ tripod screw mount. The initial design used an acrylic dome to protect the lens and camera from the environment, but it has subsequently been removed due to the excessive 


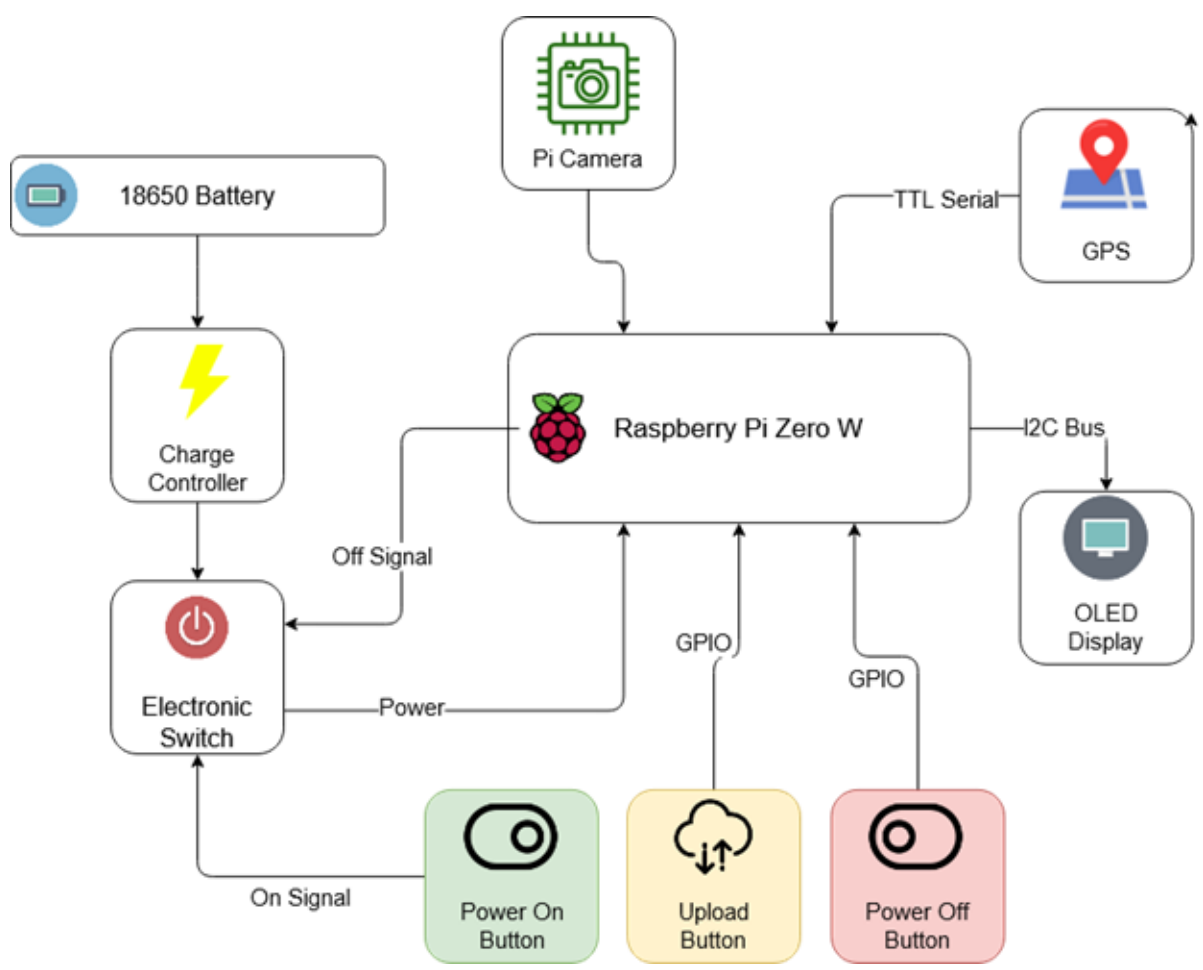

Figure 1: GONet component block diagram and functional interactions.

optical distortions it created. Although image quality was improved with the removal of the dome it does make the unit more vulnerable of the risk of damage from precipitation.

\subsection{Hardware}

Internal hardware was chosen with a preference toward inexpensive, commercially available modules that still met the cost goals of the project.

Figure 1 provides an operational overview of the components and their relationships within the camera.

A Raspberry Pi Zero W is the processor for the system and is responsible for the control, imaging, management of the user interface, and image upload tasks. The Pi Zero W is Wi-Fi and Bluetooth enabled. It also has a low power demand that optimizes imaging duration.

The camera is a standard v1 Raspberry Pi Camera Module which uses a 5MP 1/4" OV5647 Sony CMOS image sensor. The spectral response curve of the RGB sensor is shown in Figure 2. The full resolution of this sensor is $2592 \times 1944$ pixels with $1.4 \mu \mathrm{m}$ pixels. The RAW $2 \pi$ image circle averages about 1830px in diameter. After $2 \times 2$ binning in post-processing the effective resolution across the image becomes half that, $\sim 915 \mathrm{px}$ (detailed further under Data Products). The optics are an after-market $0.76 \mathrm{~mm}$ focal length M12 mount lens that provides a full $180^{\circ}$ image on the camera sensor. 


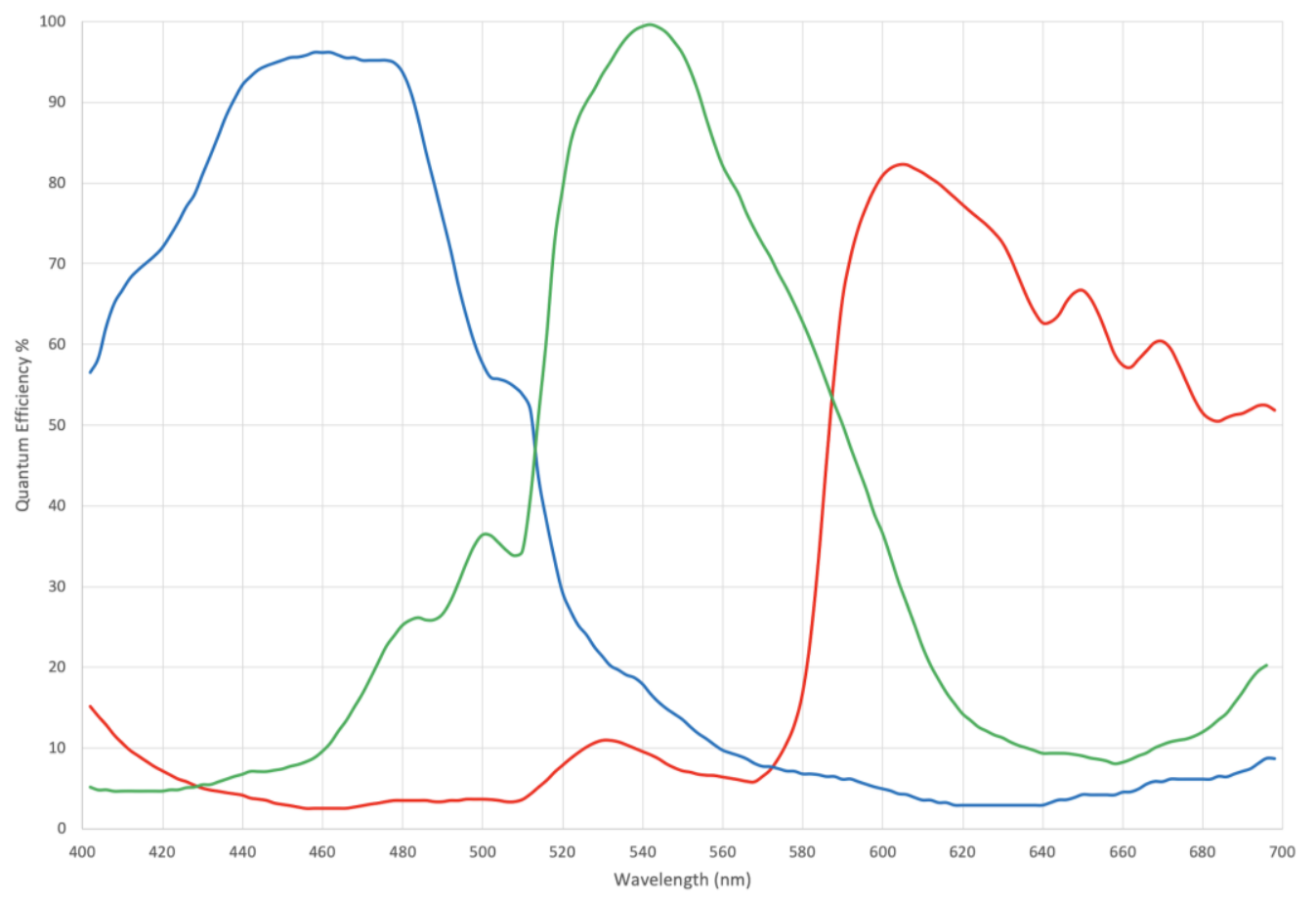

Figure 2: OV5647 sensor RGB channel spectral response curves

The only additional sensor used in the device is a U-Blox NEO-6 GPS module with a built-in antenna. The GPS provides time and location data. Once the GPS signal is acquired, the Raspberry Pi clock is synced with the GPS time. This ensures that all images are correctly timestamped. Additionally, location data from the GPS is embedded into the image metadata and included as an overlay for the JPEG preview image.

For the user interface, a monochrome 124x64 OLED screen displays the current status of the device, wireless network status, IP address, GPS status, number of images acquired, and the unique camera identifier. The unit uses a single, rechargeable and replaceable $2500 \mathrm{mAh} 18650$ battery as its power source. In field tests, this battery has been shown to provide on average 400 minutes of operation on a single charge under most conditions. In a prolonged series of tests with a single GONet camera, the average length of imaging on a single charge degraded by nearly $20 \%$ after 200 deployments totaling approximately 1,000 hours of operation.

\subsection{Software}

A GONet is operated with custom software for control, management, imaging, and user interface. It uses a Raspberry Pi Zero $\mathrm{W}$ featuring a single-core CPU with 512Mb of RAM. As the singular purpose of the device is image capture, it is important that resource consumption by the operating system itself is as minimal as possible. To minimize complexity and power consumption, installed software processes are kept to a minimum, using as many Linux-native utilities as possible needed to implement required GONet functionality. To support these requirements the following software/platforms were added to the GONet: Python, PHP, and Nginx.

The back-end control and management code is written in Python, shell script, and PHP. The GONet automates several processes including a web gallery used as a part of the user interface, Wi-Fi connection management functionality, and the primary imaging function.

Control and Management software subsystems can be broken out into the following functions: display, control (buttons), web-based gallery, scheduling, image upload, software update, Wi-Fi connection management, and repair/recovery.

The main control sub-system manages the display, button presses, image upload, and software updates, which initializes the start-up of the GONet. At that time, it will initialize the display and query the device to gather various parameters that describe the current state of the GONet, which is displayed on the OLED. 
The upload button works via a series of commands that first terminate the imaging cycle, initiates the image upload and, when completed, downloads the latest software. The GONet system uses Amazon Web Services (AWS) as the back end for image storage. Once the upload is initiated, the total number of stored images shown on the display will decrease until complete when a software update is initiated. The update process compares the unit's software version tag and automatically downloads updates if available.

\subsection{Image Storage and Management}

The backend service used for image storage and software updates is Simple Storage Service or S3, provided by AWS. S3 is a global, scalable, object-based storage service with very low cost, high availability, and reliability. Additionally, AWS provides tools that work on ARM-based Linux operating systems, like Raspbian, making it very easy to integrate with existing processes.

The large number of images captured by GONets require processing. Since the images are stored in S3 they have native integration with other AWS services, like many Machine Learning services, that can be used for sorting, classification, and analysis of captured images. While there are many advanced features and functions available with AWS, our current use to support the GONet project is minimal - image storage and software updates. Future utilization of AWS capabilities for post-processing, machine learning tasks and image analysis are being developed. Future plans include creating a web-based user interface with all the features and functionality needed to support the needs of GONet users.

\subsection{User Interface}

The GONet camera has two user interfaces: hardware and web-based. The hardware interface on the front control panel of the GONet camera consists of three pushbuttons and an OLED display (see Figure 3). The software interface is primarily meant for viewing and managing captured images.

The OLED display shows the current and overall status of the device including: Device IP address, wireless network name, device name and software version, number of images on the device, GPS status and satellites in view, and current device function. During imaging, to minimize the light output from the OLED screen, all other information is turned off and only a "Status: Imaging" message is displayed.

A web-based interface is available by logging into the GONet's IP address via a web browser. This includes an image gallery that allows for a quick, low demand method of viewing and downloading images. It also gives the end user the ability to delete images that are not needed directly from the camera before uploading. Additionally, the web interface allows for adding additional wireless networks.

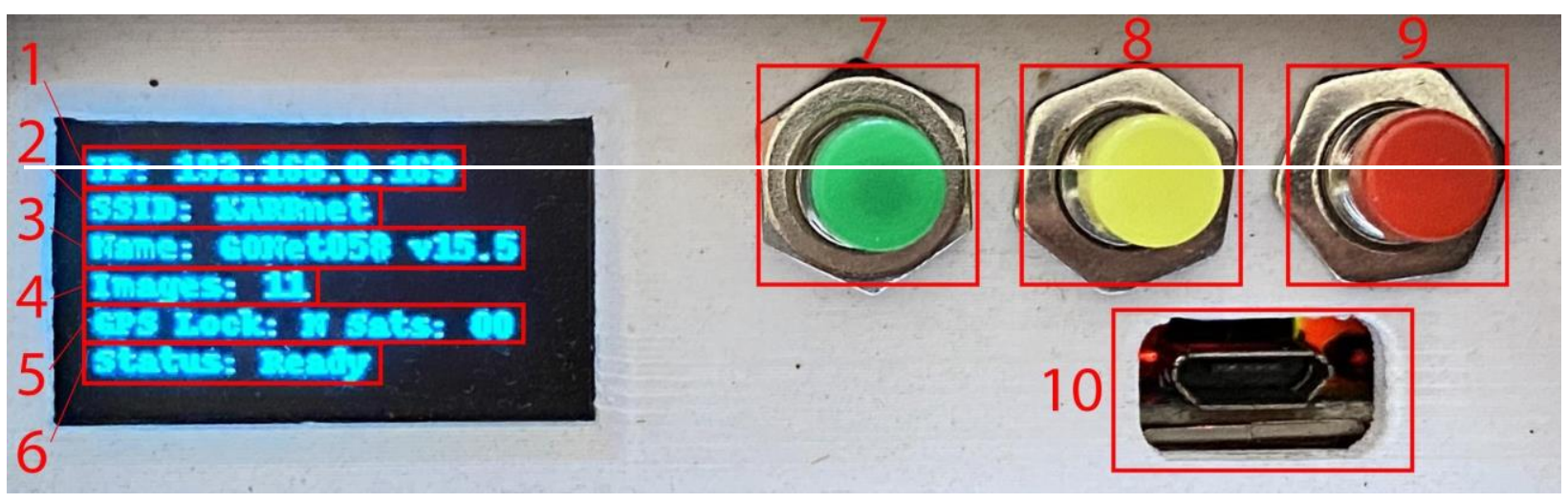

Figure 3: GONet camera control panel user interface includes (1) device IP address, (2) wireless network, (3) camera identifier and software version, (4) number of images on camera, (5) GPS lock status and visible satellites, (6) status, (7) power-on button, (8) upload button, (9) power-off button, and (10) charging port. 


\section{Operation}

From the onset of the project, one of the guiding design principles was to create a simple, user-friendly device for night-to-night use with minimal training. Three buttons are used for powering the unit on, off and for uploading collected images. Additionally, a thorough set of Standard Operating Procedures (SOPs) and manuals have been developed and are available for end-users through a shared Google Drive resource.

Each device requires access to the internet to upload captured images and download software updates. A wireless network needs to be configured for each unit. For this, the end user provides a SSID (network name) and password for the wireless network they would like to join. Wireless networks that use "captive portals", or landing pages that require users to accept terms of service and/or sign in, are not supported. This process requires removal of the four screws from the bottom of the GONet to access the SD card. A file, wpa_supplicant.conf, needs to be configured and placed on the boot volume of the SD card. Once the initial Wi-Fi configuration is made, internal access to the camera will no longer be needed except in the case of physical repairs.

Before deployment of the GONet camera, ideally the user ensures the battery is fully charged. The battery is charged using a standard micro-USB cable plugged into the front of the device. While the device is charging a blinking red light is visible through the base. Once charging is complete, the red light will remain steady, and the device is ready for deployment. A full charge may require a number of hours.

For deployment, the unit is set in the desired location - ideally with a clear view of the sky - using a tripod or a flat surface, leveled and oriented to the north. The user simply presses the green button to power on the device. We recommend performing the first cycle of images with the lens cap on to record dark frames for calibration. Within 5 minutes from power up, the device will begin its first imaging cycle. A full imaging cycle takes approximately 2.5 minutes to complete. Once the cycle is complete, Status will change to "Ready" and all the on-screen information is visible. The GONet camera will continue to image automatically every five minutes until the battery runs below operational voltage or the user intervenes.

Nighttime GONet images average 8-9 MB each and a full charge will provide between 6-7 hours of continuous operation. The camera takes a set of five, 6 second exposure images in every five-minute cycle, so deployment on a full charge will generate about $3.6 \mathrm{~GB}$ of data from approximately 80 image sets. The cameras use a $32 \mathrm{~GB}$ microSD card so, accounting for system overhead, a GONet camera can collect six to seven nights of data before requiring an upload.

Once imaging has been completed, to upload the data the GONet must be in range of a pre-configured wireless network and at least partially recharged. It is recommended that the GONet is connected to a USB power source before beginning an upload. Most home internet connections are optimized for downloads and can be very slow for uploads. Depending on the number of images captured and individual internet connection performance, upload of a single night of imaging can take a number of hours. Once the GONet has connected to a network and acquired an IP address, pressing the yellow button initiates the upload to the server. At that time, status on the display will show "Uploading". Images are uploaded in batches, several at a time, and as each batch has finished uploading, the number of images shown on the GONet will decrease. This will continue until all images are uploaded. Once the upload to the AWS server is completed, the GONet will check for any software updates. If a new version is available, it is downloaded and will install after the next reboot.

\section{Data Products}

The GONet cameras, by default, are programmed to capture a set of five images every five minutes. At every fiveminute interval the unit captures five six-second exposures in a raw format with a JPG thumbnail appended to the file. The raw image data are extracted with a custom Python script to a 2x2 pixel Bayer BGGR array, which is converted into a lossless 16-bit TIFF file (

Figure 4). Due to the initial goal of keeping the units affordable, the OmniVision 5647 camera used in the GONet camera delivers a relatively low resolution and high system noise compared to more expensive DSLR all-sky systems. Regardless of this limitation, the GONet camera can still provide useful data in many environments, except for the darkest locations. For radiance data analysis, the green channels are used as an approximation for visual spectral response. The two green channels per pixel are averaged and after the $2 \times 2$ pixel binning, the angular scale corresponds 
to about a 12 arcminute per pixel resolution at the image center. For color analysis, the full RGB information can be used.
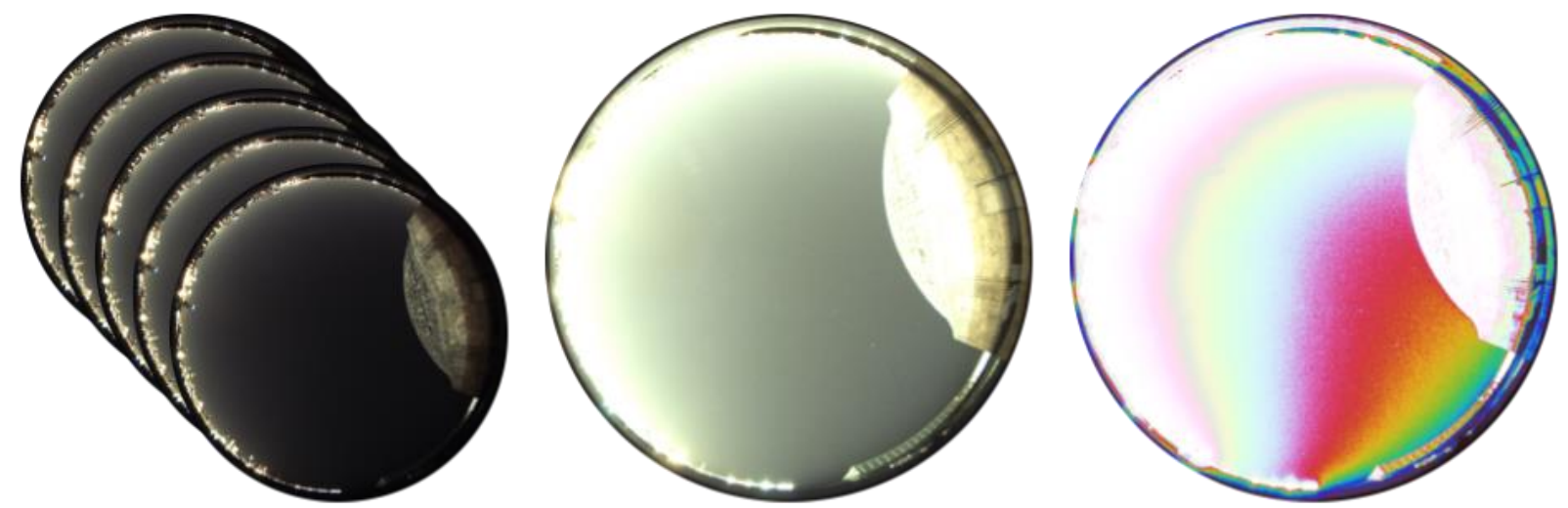

Figure 4: The GONet image processing pipeline. From each imaging cycle, 5 raw TIFFs with dark frame subtraction (left) are a combined with a summation stack (center). An 8-bit color gradient is applied (right) for a relative (uncalibrated) comparison between deployments.

The camera used in the GONet is hardware-limited to a maximum of a six-second exposure. To address this limit and reach the level of low-light sensitivity needed for proper nighttime analysis, a summation stack is performed on each set of five raw images to effectively create a 30-second exposure. By stacking, the dynamic range is also improved. Initial rounds of test observations were performed in or near the city of Chicago with a high level of ALAN. Even with the short six-second exposure, often the images neared saturation, particularly on overcast nights when the sky luminance could exceed $300 \mathrm{mcd} / \mathrm{m} 2$ (14 mag/arcsec2).

Subsequent tests in more pristine nighttime environments were able to help characterize the quality and limitations of the device (Figure 5). From these deployments, by identifying stars in the post-processed images using astrometry.net, we were able to determine the observable limit of the imaging system. A number of stars within $30^{\circ}$ of zenith of 4.3 magnitude were identified in the images and had an average pixel value of 41 ADU above local background. Assuming the linearity from lab calibration and at a scale of $790 \mathrm{arcsec} / \mathrm{px}$, the lowest average detectable signal of 8 ADU ( 1 sigma variation of the dark frames) should correspond to a $20.5 \mathrm{mag} / \mathrm{arcsec} 2$ limit for detection of sky radiance. 


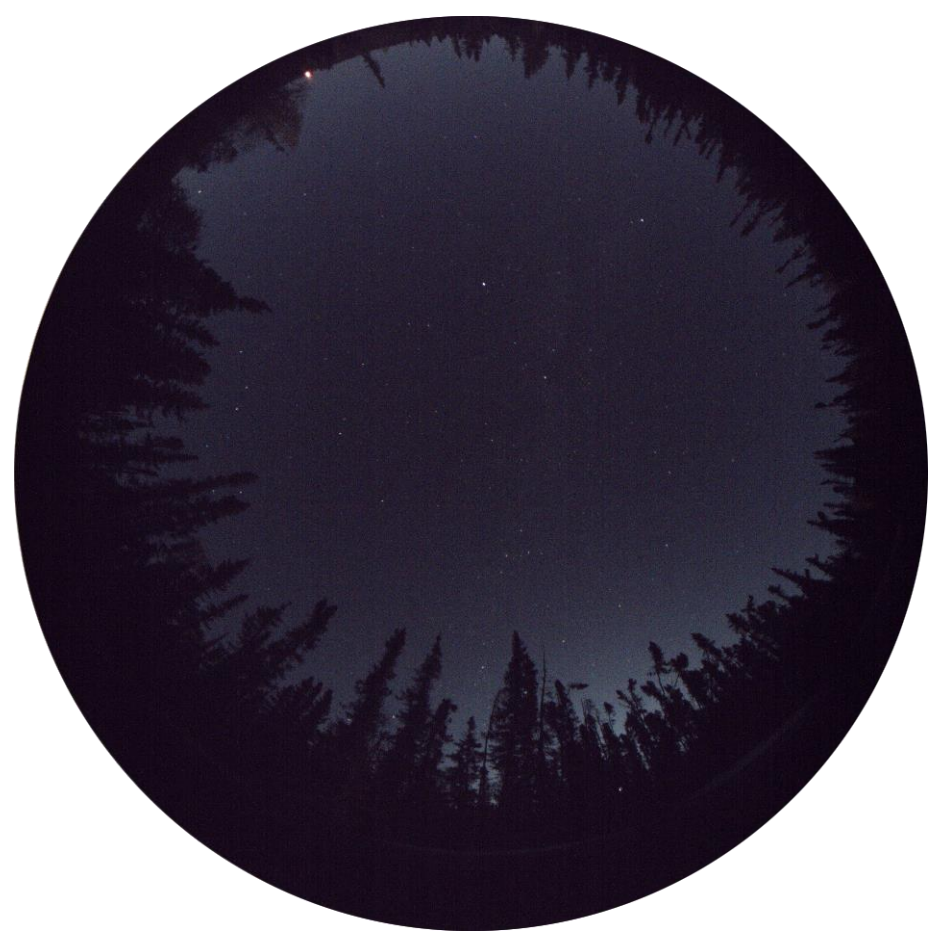

Figure 5: A stacked set of GONet images from a dark sky location (Yellowknife, Northwest Territories, Canada). Using astrometry.net, stars as faint as magnitude 4.4 were identified near the zenith.

An initial set of three GONet prototypes were calibrated and tested in the field alongside the U.S. National Park Service all-sky imaging system by Night Sky Metrics, LLC. Their results were presented to us as a report[15]. Some of the calibration processes and recommendations have been implemented into the v1 GONet camera. Full calibration - including radiance calibration - of every camera will be a difficult task given the scope and scale of this project (

Figure 6). Many of the general recommendations within the NSM report have been addressed, including the removal of the acrylic dome, increased integration time, more extensive metadata recording and elimination of internal light leaks that may impact imaging.

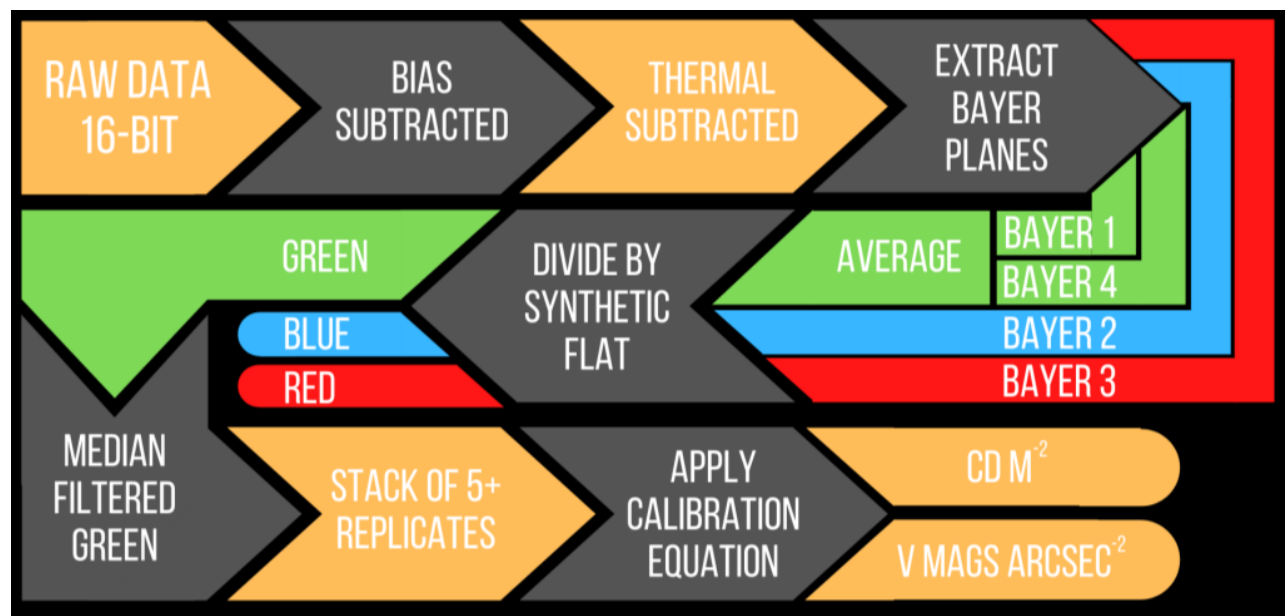

Figure 6: Flow chart of the calibration process based on the work and recommendations from NSM. To date, this process has only been fully applied to three of the GONet units 


\section{Field Results}

One unit (GONet058) has been deployed frequently overnight for nearly a year to test the reliability of the camera over an extended time period. This unit has shown no discernable degradation of image quality and minimal change in functionality. To date, GONet058 has collected over 60,000 images. An assumption of reliability has also been drawn from extensive field deployments and tests of a number of additional units; 48 operational GONets have collected over 83,000 images totaling $750 \mathrm{~GB}$ of data primarily within the Chicago area, in addition to a number of deployments in Europe and in numerous locations across North America.

A number of test deployments have been performed in and around Chicago. The first organized test deployment was performed in May 2019 in conjunction with volunteers conducting the Calling Frog Survey, a citizen science project led by herpetologists at the Chicago Academy of Sciences and Peggy Notebaert Nature Museum. Four GONet cameras were paired with audio recorders during a survey to explore whether GONet data along with sound recordings could help characterize the impact of light pollution on nocturnal frog activity. Though the results of the demonstration were merely a test of the concept, the collaboration showed promise in using GONet observations for ecological fieldwork.

A coordinated deployment of GONets was carried out in November 2019 by students participating in one of the Adler Planetarium's teen programs to obtain data simultaneously from within and outside Chicago. GONet cameras were deployed near downtown Chicago on the roof of the Adler Planetarium, while several other GONets were deployed simultaneously at Indiana Dunes National Park, $44 \mathrm{~km}$ southeast of the city center. The results demonstrate how simultaneous observations may help define the impact and form of an urban light dome on a regional scale.

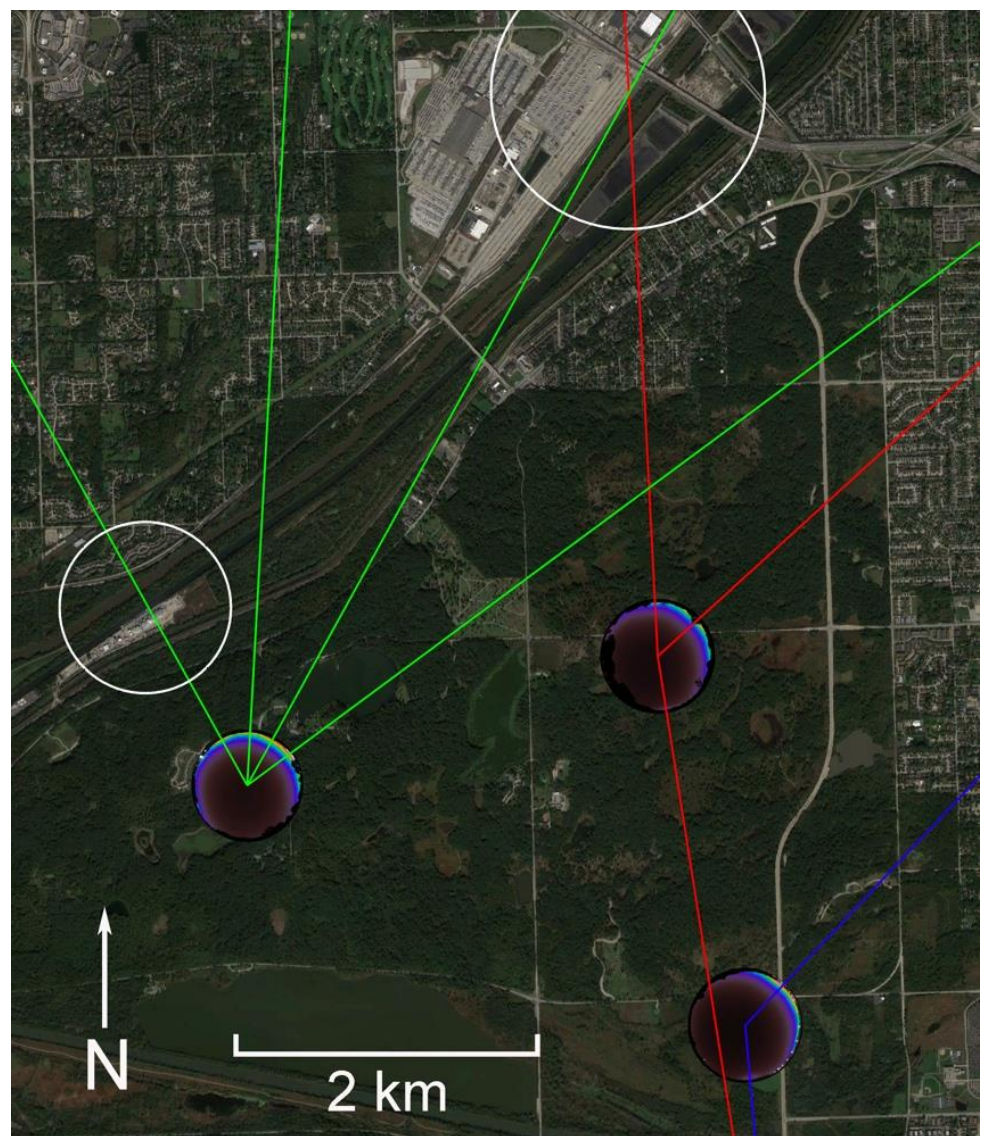

Figure 7: Simultaneous images from Mt. Forest Island forest preserve $29 \mathrm{~km}$ southeast of downtown Chicago. Light domes projected from three locations (green, red, blue) were used to identify localized sources of ALAN (white circles). Chicago is to the top left of image. (Image: Landsat/Copernicus) 
In 2020 a project using a number of GONet cameras was performed to help quantify the relative night sky quality of Mount Forest Island, an area of the Forest Preserves District of Cook County near Chicago. Mount Forest Island (MFI) is a 2,670-hectare area of public, intra-urban, protected forest land $29 \mathrm{~km}$ from downtown Chicago with almost no artificial light sources within its boundaries. Coordinated observations were made in support of an application for the area as an International Dark-Skies Association Urban Night Sky Place. On two nights in the summer of 2020, three GONet cameras were deployed simultaneously at locations within MFI and five throughout the city of Chicago.

An outcome of this observation campaign involved the GONet cameras deployed within the boundaries Mt. Forest Island. (Figure 7) The GONet cameras within MFI were separated by between 2.5 and $3.6 \mathrm{~km}$. Light domes were identified in each camera. After geolocating the images, the azimuthal center of each light dome was projected outward. With this triangulation we were able to identify their sources, confirming that some of the nearest sources (industrial and commercial facilities) had a significant impact on the nighttime skies on a very local level.

\section{Potential Applications}

Although the v1 GONet camera cannot fulfill all needs and requirements of ALAN research, there are many cases where it can be an effective research tool. A few of these potential use-cases include:

- Tracking time-dependent ALAN features throughout a night (

- Figure 8) or over extended durations;

- Large scale distributed and coordinated observations to build radiative transfer models of sky glow;

- Monitoring the color of sky glow throughout extended lighting retrofits;

- Synchronized remote-to-ground observations campaigns, and

- Low-cost, rapid monitoring of areas or conditions of interest

In addition, observations such as these may improve research efforts where it's desirable to collect measurements from multiple locations simultaneously. Surveys where a single instrument is used in succession at various locations to obtain a transect of night sky brightness, such as from urban to rural environments[2] could benefit from the potential of a distributed array of instruments.

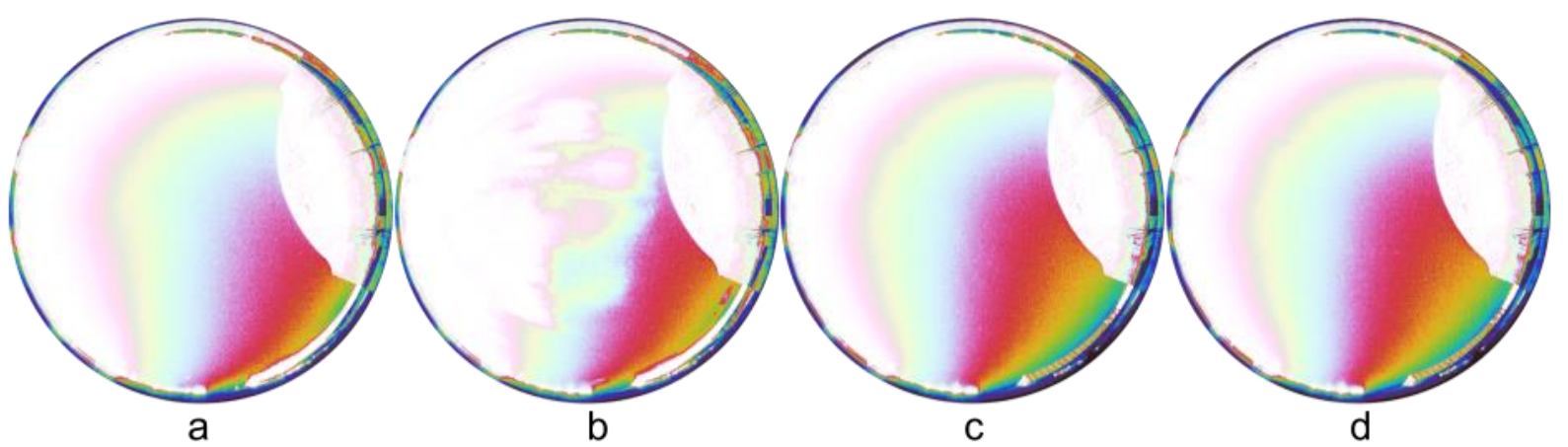

Figure 8: Example of time-dependent observations made from the Chicago lakefront in a single night at 21h (a), 23h (b), 01h (c) and 03h (d) local time. Brightening in $b$ is due to passing cloud cover. A reduction in total sky brightness can be observed by comparing the early (a) and late night $(\mathrm{c}, \mathrm{d})$ images.

\section{Discussion}

The original GONet design was intended to be a short-run prototype primarily for testing and as a student STEM project. Due to an unexpected funding opportunity, we were able to build nearly 60 units. This allowed us to use the version 1 prototype in a range of tests beyond the original scope of the project. From these tests we've identified a number of weaknesses and limitations in the original design and have planned improvements for an upgraded version 2 GONet. 
Despite the limitation of the camera at low light levels, use of the system in urban to rural environments should greatly expand on the capability of devices such as the SQM that offer a one-dimensional reading of zenith brightness. GONet cameras can be used to measure zenith brightness, generate full-sky luminance gradients, and provide rudimentary sky color information via RGB data in all but the darkest sites. Since the individual cameras are very inexpensive, this allows for deployments of multiple cameras for observations with spatial information.

Presently, user access to uploaded data is not readily available. Although all GONet data is uploaded to a central server, a user interface for accessing those data has yet to be designed. Considerations and sensitivity regarding privacy and security have slowed the development of a method of open data sharing. As of now, user access to data is on a per request basis.

One of the major weaknesses in the reliability of the initial design occurred in the electrical wiring, which resulted in frequent shorting of some units. This is being addressed in the v2 design along with many other issues noted in the following section.

\section{Version 2 Design}

Based on extensive testing of the first version GONet camera, in addition to feedback from researchers and endusers, a number of features, upgrades, and improvements are being made to a version 2 model presently in development (Figure 9).

Regarding the hardware, these improvements include: replacing the OV5647 camera with a more sensitive, higherresolution sensor that allows for longer exposures and the use of a higher quality lens; using a higher-capacity battery, which will extend imaging up to 10 hours on a single charge; a PCB (Printed Circuit Board) design to eliminate internal wiring; and an improved user interface with a higher-resolution color screen to allow more feedback and operational information.

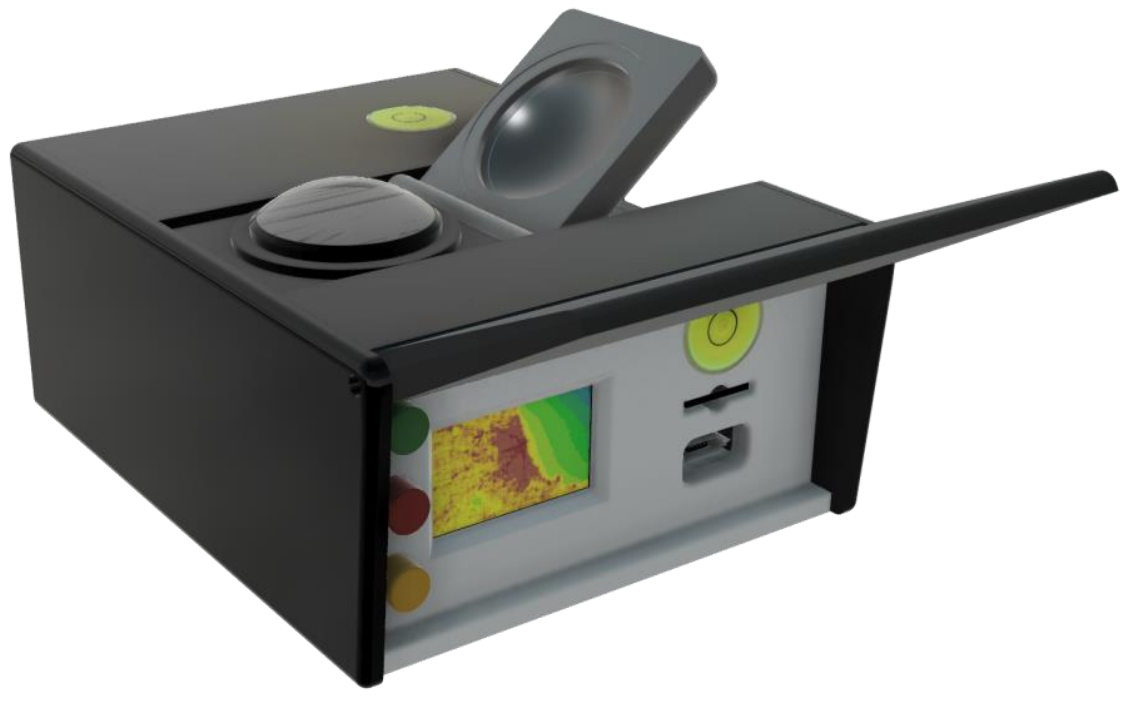

Figure 9: CAD rendering of the version 2 GONet camera in development.

Design changes include: the option to mount the camera vertically and/or have two units back-to-back for $4 \pi$ imaging; external access to the SD card to eliminate the need to open the unit for configuration and customization; and a built-in lens cover that also allows for the capture of dark frames to support calibration before each imaging deployment.

Software improvements in development include: a programmable button for custom imaging routines, such as imagin $\mathrm{g}$ on demand; a web-based user interface for camera control and customization; a server-based method for accessing, sharing, and processing data; and a more rigorous method of calibration. 


\section{References:}

[1] Duriscoe, D. M. (2016). Photometric indicators of visual night sky quality derived from all-sky brightness maps. Journal of Quantitative Spectroscopy and Radiative Transfer, 181, 33-45..

[2] Jechow, A., Kyba, C. C., \& Hölker, F. (2020). Mapping the brightness and color of urban to rural skyglow with all-sky photometry. Journal of Quantitative Spectroscopy and Radiative Transfer, 250, 106988.

[3] Zamorano, J., de Miguel, A. S., Ocaña, F., Pila-Diez, B., Castaño, J. G., Pascual, S., ... \& Nievas, M. (2016). Testing sky brightness models against radial dependency: A dense two dimensional survey around the city of Madrid, Spain. Journal of Quantitative Spectroscopy and Radiative Transfer, 181, 52-66.

[4] Ges, X., Bará, S., García-Gil, M., Zamorano, J., Ribas, S. J., \& Masana, E. (2018). Light pollution offshore: Zenithal sky glow measurements in the mediterranean coastal waters. Journal of quantitative spectroscopy and radiative transfer, 210, 91-100.

[5] Zamorano, J., García, C., Tapia, C., de Miguel, A. S., Pascual, S., \& Gallego, J. (2016). Stars4all night sky brightness photometer. International Journal of Sustainable Lighting, 18, 49-54.

[6] Sánchez de Miguel, A., Aubé, M., Zamorano, J., Kocifaj, M., Roby, J., \& Tapia, C. (2017). Sky Quality Meter measurements in a colour-changing world. Monthly Notices of the Royal Astronomical Society, 467(3), 29662979.

[7] Jechow, A., Kyba, C., \& Hölker, F. (2019). Beyond all-sky: assessing ecological light pollution using multispectral full-sphere fisheye lens imaging. Journal of Imaging, 5(4), 46.

[8] Duriscoe, D. M., Luginbuhl, C. B., \& Moore, C. A. (2007). Measuring night-sky brightness with a wide-field CCD camera. Publications of the Astronomical Society of the Pacific, 119(852), 192.

[9] Bará, S., Tapia, C. E., \& Zamorano, J. (2019). Absolute radiometric calibration of TESS-W and SQM night sky brightness sensors. Sensors, 19(6), 1336.

[10] Pun, C. S. J., So, C. W., Leung, W. Y., \& Wong, C. F. (2014). Contributions of artificial lighting sources on light pollution in Hong Kong measured through a night sky brightness monitoring network. Journal of quantitative spectroscopy and radiative transfer, 139, 90-108.

[11] Puschnig, J., Posch, T., \& Uttenthaler, S. (2014). Night sky photometry and spectroscopy performed at the Vienna University Observatory. Journal of Quantitative Spectroscopy and Radiative Transfer, 139, 64-75.

[12] Hänel, A., Posch, T., Ribas, S. J., Aubé, M., Duriscoe, D., Jechow, A., ... \& Kyba, C. C. (2018). Measuring night sky brightness: methods and challenges. Journal of Quantitative Spectroscopy and Radiative Transfer, 205, 278290.

[13] Kolláth, Z., \& Dömény, A. (2017). Night sky quality monitoring in existing and planned dark sky parks by digital cameras. arXiv preprint arXiv: 1705.09594.

[14] de Miguel, A. S., Kyba, C. C., Aubé, M., Zamorano, J., Cardiel, N., Tapia, C., ... \& Gaston, K. J. (2019). Colour remote sensing of the impact of artificial light at night (I): The potential of the International Space Station and other DSLR-based platforms. Remote sensing of environment, 224, 92-103.

[15] WALCZAK, K., CRIM, G., GESITE, T., HABTEMICHAEL, S., MORGAN, J., TARR, C., ... \& WIEDEMANN, J. The GONet (Ground Observing Network) Camera: An Inexpensive Light Pollution Monitoring System. 